\title{
A CRITERION FOR FINITE MODULE TYPE
}

\author{
CHRISTINE BESSENRODT
}

\begin{abstract}
The following result is proved: If a p-block of a finite group has only finitely many indecomposable liftable modules with the defect group of the block as a vertex or if it has only finitely many indecomposable periodic modules, then the block is of finite module type.
\end{abstract}

1. Introduction. The purpose of this paper is to give a criterion for a block to be of finite module type (that is, the block has only finitely many nonisomorphic indecomposable FG-modules) by looking at some nice families of modules in the block, namely the liftable and the periodic modules.

Let $G$ be a finite group and $(F, R, K)$ a $p$-modular splitting system for $G$, that is, $R$ is a complete discrete valuation ring with quotient field $K$ of characteristic 0 and residue class field $F$ of characteristic $p$, such that $K$ and $F$ are splitting fields for all subgroups of $G$. For further notation we refer to [3]. Then we have the following criterion:

THEOREM. Let $B$ be a block of FG with defect group D. Then the following are equivalent:

(i) $B$ is of finite module type.

(ii) $B$ has only finitely many indecomposable liftable modules with vertex $D$.

(iii) $B$ has only finitely many indecomposable periodic modules.

Of course, "finitely many" always means up to isomorphism.

2. Preliminary results on periodicity. In the following $A \in\{R, F\}$ and for $A=R$ an $A G$-module means an $R G$-lattice, that is an $R G$-module which is finitely generated and torsionfree as an $R$-module. Moreover, in this chapter $F$ and $K$ need not be splitting fields.

First let us recall the definition of Heller's operator $\Omega$. If $U$ is an $A G$-module then by $[4,6]$ there exists a minimal projective presentation $0 \rightarrow \Omega U \rightarrow P \rightarrow U \rightarrow 0$, that is, $P$ is a projective $A G$-module and $\Omega U$ has no projective summands. $P$ and $\Omega U$ are uniquely determined by $U$ (up to isomorphism), and if $U$ is indecomposable and nonprojective, then so is $\Omega U$. By iterating this process we get $\Omega^{i} U$, for all $i \in \mathbf{N}$. Then an $A G$-module $U$ is called periodic if $U \simeq \Omega^{i} U \oplus L$ for some $i>0$ and projective $A G$-module $L$.

A block $B$ of $A G$ is called periodic if all modules in $B$ are periodic.

Received by the editors January 19, 1982.

1980 Mathematics Subject Classification. Primary 16A26, 20 C05.

Key words and phrases. Block, liftable module, periodic module, defect group, vertex.

(C)1982 American Mathematical Society $0002-9939 / 82 / 0000-1122 / \$ 0.150$ 
2.1 REMARK. Since a direct sum of modules is periodic iff all summands are periodic, a block $B$ is periodic iff the indecomposable modules in $B$ are periodic.

The following fact is well known and can easily be proved.

2.2 LEMMA. An AG-module is periodic iff its source is periodic.

2.3 Proposition. $A$ block $B$ of $A G$ is periodic iff its defect group $D$ is cyclic or generalized quaternion.

Proof. If $A=F$ then by [2] there is an indecomposable $F G$-module $U$ in $B$ with source $F_{D}$. If $A=R$, then using this for the corresponding block of $F G$ and [8] we thus also have an indecomposable $R G$-lattice $U$ in $B$ with source $R_{D}$. Now if $B$ is periodic then so is $U$ and hence $A_{D}$ by 2.2. But this is well known to be equivalent to $D$ being cyclic or generalized quaternion. If $D$ is cyclic or quaternion then all $A D$-modules are periodic. Hence all modules in $B$ are direct summands of induced periodic modules, and so $B$ is periodic.

\section{Proof of the theorem.}

3.1 Proposition. Let $F$ be any field of characteristic $p$ and $B$ be a block of $F G$ with defect group $D$, which has only finitely many indecomposable liftable FG-modules with vertex $D$. Then $B$ is periodic.

Proof. As in the preceding proof there is an indecomposable module $M$ in $B$ with source $F_{D}$ which is liftable, say $M \simeq \bar{U}$, where $U$ is an indecomposable $R G$-lattice. Now $\Omega^{i}(M) \simeq \overline{\Omega^{i}(U)}$ for all $i \in \mathbf{N}_{0}$ by [4], so the $\Omega^{i}(M)$ are indecomposable liftable modules with vertex $D$ in $B$. By assumption there are only finitely many such modules in $B$, hence $M$ must be periodic and by 2.2 so is $F_{D}$. Now this implies $B$ is periodic by 2.3 .

3.2 Proposition. Let $B$ be a block with generalized quaternion defect group $Q$. Then $B$ has infinitely many indecomposable liftable modules with vertex $Q$.

Proof. Let $t$ be the unique involution in $Q$. First suppose $\langle t\rangle \triangleleft G$. Then $G=C_{G}(\langle t\rangle)$. By $[3,64.5]$ we have a uniquely determined block $\bar{B}$ of $\bar{G}=G /\langle t\rangle$ corresponding to $B$ (by $\bar{B} \subseteq B$ ) with defect group $Q /\langle t\rangle$. Since $Q /\langle t\rangle$ is neither cyclic nor quaternion, 2.3 together with 3.1 shows that $\bar{B}$ has infinitely many indecomposable liftable modules with vertex $Q /\langle t\rangle$. By regarding these $F \bar{G}$-modules as $F G$-modules we obtain an infinite number of indecomposable liftable modules with vertex $Q$ in $B$.

In the general case we use Green correspondence between $N_{G}(\langle t\rangle)$ and $G$. As $\langle t\rangle$ is a trivial intersection set, the Green correspondent of a liftable module is again liftable (this is easy, see e.g. [7, 2.8]). So by using the first case and Green correspondence we get an infinite set of nonisomorphic indecomposable liftable modules with vertex $Q$ in $B$.

3.3 PROOF OF THE THEOREM.

(i) $\Rightarrow$ (ii), (iii) is trivial.

(ii) $\Rightarrow$ (i) As a direct consequence of the preceding propositions $D$ is cyclic; hence $B$ is of finite module type by [5]. 
(iii) $\Rightarrow$ (i) By [5] a block is of finite module type iff its defect group is cyclic. So suppose $D$ is not cyclic. Then by [1] there are infinitely many indecomposable periodic $F D$-modules. Hence there are infinitely many indecomposable periodic $F Y$-modules which are sources, for some $Y \leqslant D$, since a source is a source for only finitely many $F D$-modules. By [2] each of these sources is a source for an indecomposable module in $B$, which is periodic by 2.2 . Since every module has only finitely many sources we thus have infinitely many indecomposable periodic modules in $B$.

\section{REFERENCES}

1. J. L. Alperin, Periodicity in groups, Illinois J. Math. 21 (1977), 776-783.

2. D. W. Burry, The distribution of modular representations into blocks, Proc. Amer. Math. Soc. 78 (1980), 14-16.

3. L. Dornhoff, Group representation theory B, Marcel Dekker, New York, 1971.

4. J. A. Green, Vorlesungen über modulare Darstellungstheorie endlicher Gruppen, Vorlesungen aus dem Math. Institut Giessen, 1974.

5. W. Hamernik, Indecomposable modules with cyclic vertex, Math. Z. 142 (1975), 87-90.

6. A. Heller, Indecomposable representations and the loop space operation, Proc. Amer. Math. Soc. 12 (1961), 640-643.

7. R. M. Peacock, Ordinary character theory in a block with a cyclic defect group, J. Algebra 44 (1977), 203-220.

8. L. L. Scott, Modular permutation representations, Trans. Amer. Math. Soc. 175 (1973), 101-122.

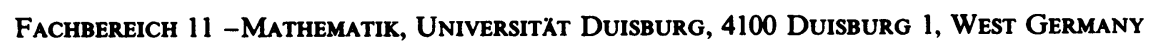

